\title{
Criticality in confined ionic fluids
}

\author{
J. E. Flores-Mena, ${ }^{1,2}$ Marcia C. Barbosa, ${ }^{2,3}$ and Yan Levin ${ }^{2, *}$ \\ ${ }^{1}$ Facultad de Ciencias de la Electrónica, Universidad Autónoma de Puebla, AP J-48, Puebla 72570, Puebla, Mexico \\ ${ }^{2}$ Instituto de Fisica, Universidade Federal do Rio Grande do Sul, Caixa Postal 15051, 91501-970 Porto Alegre (RS), Brazil \\ ${ }^{3}$ Center of Polymer Studies, Boston University, Boston, Massachusetts 02215
}

(Received 15 January 2001; published 15 May 2001)

\begin{abstract}
A theory of a confined two-dimensional electrolyte is presented. The positive and negative ions, interacting by a $1 / r$ potential, are constrained to move on an interface separating two solvents with dielectric constants $\epsilon_{1}$ and $\epsilon_{2}$. It is shown that the Debye-Hückel type of theory predicts that this two-dimensional Coulomb fluid should undergo a phase separation into a coexisting liquid (high-density) and gas (low-density) phases. We argue, however, that the formation of polymerlike chains of alternating positive and negative ions can prevent this phase transition from taking place.
\end{abstract}

DOI: 10.1103/PhysRevE.63.066104

PACS number(s): 05.70.Fh, 64.70.-p, 64.60.-i

\section{INTRODUCTION}

Over the last decade the need to understand Coulombic criticality has provided a new impetus to the study of electrolyte solutions. The current wave of exploration can be traced to the pioneering experiments of Pitzer and Singh, who have first reported a surprising finding that Coulomb interactions might belong to the mean-field universality class [1]. This suggestion has not gone unchallenged and, in fact, later experiments are consistent with a crossover from meanfield to Ising universality class very close to the critical point [2]. The crossover, if indeed it exists, is much closer to the critical point than for any other known fluid.

From the theoretical point of view it is very hard to justify anything but Ising criticality $[3,4]$. The goal for the theorists must then lie in a seemingly simpler task of finding why the crossover region for electrolytes is so narrow. Unfortunately even this program has failed to produce any satisfactory explanation [5]. Most calculations suggest that the critical region for electrolytes should be comparable to that of a Lennard-Jones fluid. To further confound the mystery, Monte Carlo simulations are once again pointing in the direction of mean-field criticality [6].

A seemingly unrelated problem concerns the disappearance of the anticipated liquid-gas transition in a system of dipolar hard spheres (DHS). Since the DHS is the simplest realization of a polar fluid, for a long time it has been believed that it must exhibit a liquid-gas phase separation. It came, therefore, as quite a surprise when the Monte Carlo simulations failed to locate this singularity [7]. Instead what they found was that as the temperature was lowered, the dipolar particles aligned, forming polymerlike chains. Since these chains interact weakly [8], it has been argued that the liquid-gas transition must be driven entirely by the free unassociated dipoles [9]. In fact a critical density of free dipoles is needed for the phase transition to occur. At low temperatures, where most of the theories localize the transition, the formation of dipolar chains depletes the density of

*Corresponding author: Email address: levin@if.ufrgs.br free dipoles below the critical threshold necessary for the transition to take place [9].

In this paper we shall study a third model, which we hope might span the gap between the three-dimensional Coulomb gas and the dipolar hard spheres, and thus shed some additional light on the criticality in these interesting and important systems. Our new model consists of a neutral electrolyte confined to a two-dimensional plane [10]. This can be visualized as oppositely charged surfactant molecules adsorbed to a water-oil interface. An example of such a system is cetyltrimethyl amonium-hydroxy naphthalene carboxylate, which is composed of two surface active parts, $\mathrm{CTA}^{+}$and $\mathrm{HNC}^{-}$[11].

We shall argue that unlike the $3 d$ electrolyte [12], the confined $2 d$ plasma might not phase separate. Instead, as the temperature is lowered, chains composed of alternating positive and negative ions will begin to form $(\ldots+-+$ - ... ) [4]. Just as with dipolar chains, these new clusters interact weakly between themselves. However, they diminish the concentration of free ions below the critical value necessary for the transition to take place.

\section{MODEL}

Our system consists of an ionic fluid of $N_{+}$positive and

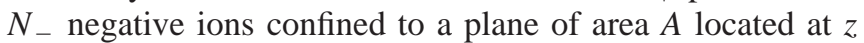
$=0$, separating two different solvents occupying the halfspaces at $z>0$ and $z<0$. We shall restrict our attention to the neutral electrolytes, for which $N_{+}=N_{-}=N / 2$. The solvents are treated as uniform mediums with dielectric constants $\epsilon_{2}$ and $\epsilon_{1}$ for $z>0$ and $z<0$, respectively. The ions of both species are modeled as hard spheres of diameter $a$ and charge $\pm q$ located at the center. It will be convenient to define the dimensionless particle density as $\rho_{ \pm}^{*}=\rho_{ \pm} a^{2}$, where $\rho_{i}$ $=N_{i} / A$. The total density of ions is $\rho=\rho_{+}+\rho_{-}$.

All the relevant thermodynamic information is contained in the free-energy density, $f=F / A$. Unfortunately due to the complexity of interactions, no exact expression for $f$ can be found. We shall, therefore, attempt to construct the approximate free energy using the most relevant contributions. These can be divided into entropic and electrostatic, 


$$
f\left(T, \rho_{+}, \rho_{-}\right)=f_{e n t}^{(1)}\left(T, \rho_{+}, \rho_{-}\right)+f_{e l}\left(T, \rho_{+}, \rho_{-}\right) .
$$

The entropic (mixing) free energy is given by

$$
\begin{aligned}
\beta f_{\text {ent }}^{(1)}\left(T, \rho_{+}, \rho_{-}\right)= & {\left[\rho_{+} \ln \left(\rho_{+} \Lambda^{2}\right)-\rho_{+}\right.} \\
& \left.+\rho_{-} \ln \left(\rho_{-} \Lambda^{2}\right)-\rho_{-}\right]
\end{aligned}
$$

where $\Lambda=\sqrt{2 \pi \hbar^{2} / m k_{B} T}$ is the thermal de Broglie wavelength of the ions. The second term in Eq. (1) is due to electrostatic interactions. It is important to note that the electrostatic free energy is purely correlational, since the meanfield contribution is zero. To calculate $f_{e l}$, let us fix one ion at the origin. Adopting the cylindrical coordinate system $(\varrho, \varphi, z)$, the central ion is located at $\varrho=0, z=0$. Due to the electrostatic interactions the other particles will arrange themselves within the plane in accordance with the Boltzmann distribution. Since no charge is present in the regions $z>0$ and $z<0$, the electrostatic potential there must satisfy the Laplace equation. Appealing to the azimuthal symmetry and taking into account the fact that the potential should vanish at infinity, we find [13]

$$
\phi_{1}(\varrho, z)=\int_{0}^{\infty} A_{1}(k) J_{0}(k \varrho) e^{k z} d k \quad \text { for } \quad z<0
$$

and

$$
\phi_{2}(\varrho, z)=\int_{0}^{\infty} A_{2}(k) J_{0}(k \varrho) e^{-k z} d k \text { for } \quad z>0
$$

where $J_{0}(x)$ is the Bessel function of order zero.

The functions $A_{1}(k)$ and $A_{2}(k)$ are determined by the boundary conditions; continuity of electrostatic potential,

$$
\phi_{2}(\varrho, 0)=\phi_{1}(\varrho, 0)
$$

and discontinuity of displacement field across the $z=0$ plane,

$$
\left[\epsilon_{2} \mathbf{E}_{2}(\varrho, z)-\epsilon_{1} \mathbf{E}_{1}(\varrho, z)\right] \cdot \hat{n}=4 \pi \sigma_{e f f}(\varrho)
$$

where $\sigma_{e f f}(\varrho)$ is the surface charge density and $\hat{n}$ is a unit vector normal to the interface, pointing from region 1 to 2 . The continuity of electrostatic potential results in $A_{1}(k)$ $=A_{2}(k)=A(k)$, while Eq. (6) requires that

$$
2 \int_{0}^{\infty} k A(k) J_{0}(k \varrho) d k=\frac{4 \pi \sigma_{e f f}(\varrho)}{D}
$$

where $D=\left(\epsilon_{1}+\epsilon_{2}\right) / 2$. The surface charge density [13] is given by

$$
\sigma_{e f f}(\varrho)=\sigma_{s}(\varrho)+\frac{q \delta(\varrho)}{2 \pi \varrho}
$$

The term $\sigma_{s}$ is the charge density of the "ionic cloud" around the central ion,

$$
\sigma_{s}(\varrho)=q \rho_{+} e^{-\beta q \phi}-q \rho_{-} e^{+\beta q \phi} .
$$

Equations (7) and (8) are only valid in the limit $a \rightarrow 0$. Unfortunately it is highly nontrivial to take into account the boundary condition associated with the finite ionic size. To circumvent this difficulty, we shall first solve all the equations in the point particle limit. Then to account for the finite particle size, we shall replace the bare charge of the central ion $q$, in Eq. (8), by an effective charge $Q, q \rightarrow Q$. The effective charge $Q$ will be determined by the condition of an overall charge neutrality

$$
2 \pi \int_{a}^{\infty} \sigma_{s}(\varrho) \varrho d \varrho=-q
$$

For the $3 d$ electrolyte this procedure leads to an electrostatic potential identical with the one found from the exact solution of the Debye-Hückel equations with the appropriate hard-core boundary conditions [14]. In the present geometry the procedure outlined above will only be an approximation, although we believe a rather good one.

In the spirit of the Debye-Hückel theory [14] we shall now linearize the Boltzmann factor in Eq. (9). The surface charge density then reduces to

$$
\sigma_{s}(\varrho)=-\frac{D \kappa_{s} \phi(\varrho)}{2 \pi}
$$

where $\kappa_{s}=2 \pi \rho^{*} / T^{*} a$ is the inverse Gouy-Chapman length and $T^{*}=k_{B} T D a / q^{2}$ is the reduced temperature. Equation (7) can now be solved yielding the expression for the electrostatic potential

$$
\phi(\varrho, z)=\frac{Q}{D} \int_{0}^{\infty} \frac{k}{k+\kappa_{s}} J_{0}(k \varrho) e^{-k|z|} d k
$$

For $z=0$, the in-plane potential agrees with the one obtained by Velazquez and Blum [10] and can be conveniently rewritten as

$$
\phi_{>}(\varrho)=\frac{Q \tau_{0}\left(\kappa_{s} \varrho\right)}{D \varrho} .
$$

The subscript " $>$ ", is included to stress that for rigid particles this form will be appropriate only outside the hard-core exclusion region, $\varrho>a$. The function $\tau_{\nu}(x)$ is defined as $[10]$

$$
\tau_{\nu}(x)=1-\frac{\pi x^{1-\nu}}{2}\left[H_{\nu}(x)-N_{\nu}(x)\right]
$$

where $H_{\nu}(x)$ and $N_{\nu}(x)$ are the Struve and the Bessel functions of order $\nu$, respectively [15]. The charge neutrality, Eq. (10), together with Eqs. (11) and (13), determines the effective charge $Q$

$$
Q=-\frac{q}{\kappa_{s} a \tau_{1}\left(\kappa_{s} a\right)} .
$$

In the limit of $a \rightarrow 0$, the renormalized charge reduces to the bare charge $q$. 
To obtain the electrostatic free energy, we require the potential inside the excluded volume region, $\phi_{<}(\varrho)$. Unfortunately the procedure presented above leaves this undetermined. The only statement that we can make is that $\phi_{<}(\varrho)$ must be of the form

$$
\phi_{<}(\varrho)=\frac{q}{D \varrho}+C(\varrho)
$$

For a three-dimensional unconstrained electrolyte, $C$ is a constant. This, however, is not the case in the present geometry and $C(\varrho)$ is a function of position. In particular the value $C(0)$ is the potential that the central ion feels due to the presence of other particles. In their earlier study, Velasquez and Blum [10] approximated $C(\varrho)$ by a constant, which they then determined by requiring continuity of electrostatic potential across the exclusion boundary $\phi_{<}(a)$ $=\phi_{>}(a)$. This, however, is a very rough approximation since there is nothing to prevent $C(\varrho)$ from being a very strongly varying function of position. To avoid this difficulty we shall use an alternate method of obtaining the value of $C(0)$. To this end we note that the potential at the center of a circularly symmetric charge distribution is

$$
C(0 ; \rho)=\frac{2 \pi}{D} \int_{a}^{\infty} \sigma_{s}(\varrho) d \varrho,
$$

where in order to emphasize that the potential depends on the density of ions, we have explicitly included $\rho$ in its definition. Using Eq. (11) with the potential given by Eq. (13) we find

$$
C(0 ; \rho)=\frac{q}{D a \tau_{1}\left(k_{s} a\right)} \int_{\kappa_{s} a}^{\infty} \frac{\tau_{0}(z)}{z} d z .
$$

The excess chemical potential can now be calculated straight forwardly by appealing to the Güntelberg charging process [16]. We find $\mu_{ \pm}^{e x}=q C(0 ; \rho) / 2$. The chemical potential of the positive and negative ions is

$$
\beta \mu_{ \pm}=\frac{\partial f}{\partial \rho_{ \pm}}=\ln \left(\frac{\rho \Lambda^{2}}{2}\right)+\frac{\beta q C(0 ; \rho)}{2} .
$$

The critical point is determined by the conditions, $\partial \mu_{ \pm} / \partial \rho$ $=0$ and $\partial^{2} \mu_{ \pm} / \partial \rho^{2}=0$, which reduce to

$$
\begin{aligned}
& 2 T^{*} \tau_{1}^{2}+I \tau_{1}-I \tau_{0}-\tau_{1} \tau_{0}=0, \\
& I \tau_{1}^{2}-4 I \tau_{0} \tau_{1}-3 \tau_{0} \tau_{1}^{2}+I \tau_{0}^{2}+2 \tau_{0}^{2} \tau_{1}+x^{2} I \tau_{1}^{2}+I \tau_{1}+x^{2} \tau_{1}^{3}+\tau_{1}^{2} \\
& \quad=0
\end{aligned}
$$

with

$$
I(x)=\int_{x}^{\infty} \frac{\tau_{0}(z)}{z} d z
$$

where $x=\kappa_{s} a$. Solving Eqs. (20), the critical point is located at $T_{c}^{*}=0.0517386$ and $\rho_{c+}^{*}=\rho_{c-}^{*}=0.00121988$.

\section{DEBYE-HÜCKEL-BJERRUM APPROXIMATION}

Clearly the low temperature at which the critical point is located should make us worry about the approximations which have been adopted. Certainly at such extreme conditions the linearization of the Boltzmann factor, Eq. (11), is no longer valid. Fortunately, all is not lost. Evidently, linearization of the Boltzmann factor in Eq. (9) diminishes the weight of configurations in which the oppositely charged ions are in a close proximity. It is possible, therefore, to correct for the omitted nonlinearities by explicitly allowing for the formation of "clusters." These clusters are assumed to be in a chemical equilibrium with the free unassociated ions, and their density is determined by the law of mass action [17,18]. The most basic such cluster is a dipole formed by a $(+-)$ pair. Within the simplest version of this theory the dipoles are treated as a noninteracting ideal specie. For the $3 d$ electrolyte this Debye-Hückel-Bjerrum approximation $(\mathrm{DHBj})[3,4]$ has proven extremely successful, predicting the location of the critical point in close agreement with the Monte Carlo simulations [12].

The total density of ions $\rho$ can then be subdivided into that of free unassociated monopoles $\rho_{1}=\rho_{+}+\rho_{-}$, and of dipolar pairs $\rho_{2}$, with $\rho=\rho_{1}+2 \rho_{2}$. In the spirit of DHBj theory we shall first treat the dipoles as ideal noninteracting entities whose concentration is determined by the law of mass action, $\mu_{+}+\mu_{-}=\mu_{2}$. The Helmholtz free-energy density is then given by

$$
\beta f_{D H B j}=f_{e n t}^{(2)}\left(\rho_{1}, \rho_{2}, T\right)+f_{e l}\left(\rho_{1}, T\right),
$$

where the entropic contribution is

$$
\begin{aligned}
\beta f_{\text {ent }}^{(2)}\left(\rho_{1}, \rho_{2}, T\right)= & {\left[\rho_{2} \ln \left(\frac{\rho_{2} \Lambda^{4}}{\xi_{2}}\right)-\rho_{2}\right] } \\
& +\left[\rho_{1} \ln \left(\frac{\rho_{1} \Lambda^{2}}{2}\right)-\rho_{1}\right] .
\end{aligned}
$$

The $\xi_{2}$ is the internal partition function of a dipolar pair

$$
\xi_{2}(T ; R)=2 \pi \int_{a}^{R} e^{-\beta U_{2}(\varrho)} \varrho d \varrho
$$

where $\beta U_{2}=a / T^{*} \varrho$ is the electrostatic potential between the associated ions. In order to evaluate $\xi_{2}(T ; R)$ we must define the distance $R$ at which two ions can be considered to be associated. Following Bjerrum [17] we choose the value of $R_{B j}$ at which $\xi_{2}(T ; R)$, as a function of $R$, has an inflection point $R_{B j}=a / T^{*}$ [4]. With this, the integral in Eq. (24) can be evaluated to yield

$$
\xi_{2}=\frac{\pi a^{2} e^{b}}{b}\left\{b^{3} e^{-b}[E i(b)-E i(1)+2 e]-b(1+b)\right\},
$$

where $b=1 / T^{*}$ and $E i(x)$ is the exponential integral function.

Since within the DHBj approximation the dipoles are treated as ideal, the electrostatic free energy only depends on 
the density of free monopoles $\rho_{1}$. Substituting the free energy into the law of mass action, the density of dipoles is given by

$$
\rho_{2}^{*}=\xi_{2} \rho_{+} \rho_{-} e^{\beta\left(\mu_{+}^{e x}+\mu_{-}^{e x}\right)} .
$$

The charge neutrality requires that $\rho_{+}=\rho_{-}=\rho_{1} / 2$. The expression for excess chemical potential was calculated in Sec. II, $\mu_{ \pm}^{e x}=\beta q C\left(0 ; \rho_{1}\right) / 2$, and the inverse Gouy-Chapman length is now $\kappa_{s}=2 \pi \rho_{1}^{*} / T^{*} a$.

Within the DHBj approximation the dipoles are treated as ideal specie, therefore, they cannot influence the location of the critical point. Thus, the critical temperature must still be $T_{c}^{*}=0.0517386$ while the critical density of monopoles must remain $\rho_{1 c}^{*}=0.00243975$. Substituting these values into Eq. (26), we find that the density of dipoles is $\rho_{2 c}^{*}$ $=1.08847$, which is extremely high. If there are so many dipoles is it also not possible that there will be higher-order clusters as well?

\section{LINEAR IONIC CHAINS}

Unfortunately as soon as we get to clusters of three ions the calculations get extremely difficult. The basic problem is the internal partition function of the higher-order clusters, which can no longer be evaluated exactly. Furthermore, while it is evident that for a cluster of three ions the lowtemperature configurations are chainlike, $(+-+)$ or $(-+-)$, this is far from obvious for a neutral cluster of two positive and two negative ions. The entropy favors a chainlike configuration $(+-+-)$, while the energy favors a compact square cluster. Which will gain in the critical region is hard to say. We note, however, that exactly the same situation was encountered for dipolar hard spheres $[9,19]$. In that case, in the vicinity of the critical point, the chainlike configurations dominated. Since it is much easier to study the linear clusters they, therefore, will provide a starting point for our analysis.

We begin by supposing that at low temperatures our system will be composed of monopoles of density $\rho_{1}$ and chains of $n$ monomers with densities $\rho_{n}$. Once again in the spirit of Bjerrum we shall first treat the clusters as ideal noninteracting species. The particle conservation requires that

$$
\rho=\sum_{n=1}^{\infty} n \rho_{n}
$$

Consider an alternating chain composed of $t$ positive and $s=n-t$ negative ions. The partition function for such a cluster is

$$
\xi_{n}(T)=\frac{1}{s ! t !} \frac{1}{A} \int_{\Omega_{n}} \prod_{i=1}^{n} d^{2} \mathbf{r}_{i} e^{-\beta U_{n}},
$$

where $\Omega_{n}$ is the configurational volume and $U_{n}$ is the total energy of interaction between the ions forming a chain

$$
U_{n}\left(\mathbf{r}_{1}, \mathbf{r}_{2}, \ldots, \mathbf{r}_{n}\right)=\sum_{i<j}^{n} \varphi_{i j}\left(r_{i j}\right)
$$

At zero temperature chains are rigid and the particles are in contact with one another. The displacement vector between two ions $i$ and $j$ is $\vec{r}_{i, j}^{0} \equiv a|i-j| \hat{x}$, where $\hat{x}$ is the unit vector along the chain. The electrostatic energy of interaction between the ions of the chain can be evaluated exactly yielding

$$
U_{n}^{0}=\frac{q^{2}}{D a} S(n)
$$

where

$$
S(n)=\sum_{k=1}^{n-1} \frac{(-1)^{k}(n-k)}{k}
$$

For nonzero but small temperatures, deviations from $\vec{r}_{i, j}^{0}$ occur. These fluctuations can be taken into account by making a Taylor expansion of $U_{n}$ around the ground state up to quadrupolar order

$$
\begin{aligned}
U_{n}= & U_{n}^{0}-\frac{q^{2}}{2 D} \sum_{i \neq j}^{n}(-1)^{i+j}\left\{\frac{\left(\vec{r}_{i j}-\vec{r}_{i j}^{0}\right)_{x}}{\left(\vec{r}_{i j}^{0}\right)^{2}}+\frac{\left(\vec{r}_{i j}-\vec{r}_{i j}^{0}\right)_{y}^{2}}{2\left|\vec{r}_{i j}^{0}\right|^{3}}\right. \\
& \left.-\frac{\left(\vec{r}_{i j}-\vec{r}_{i j}^{0}\right)_{x}^{2}}{\left|\vec{r}_{i j}^{0}\right|^{3}}\right\} .
\end{aligned}
$$

Here the subscripts $x$ and $y$ represent the components along the chain's direction and perpendicular to it, respectively. Since we are assuming small deviations from the ground state, for each ion $i$ we shall consider only fluctuation contributions to $\xi_{n}$ arising from the interactions between the nearest and the second-nearest neighbors. Choosing as the unit bases $\hat{x}$ and $\hat{y}$, vectors parallel and perpendicular to the direction of the chain in the plane $z=0$, the nearest and the second nearest displacement vectors can be written as

$$
\vec{R}_{1}^{(i)}=a\left(1+\lambda_{i}\right)\left(\begin{array}{c}
\cos \phi_{i} \\
\sin \phi_{i}
\end{array}\right)
$$

and

$$
\vec{R}_{2}^{(i)}=a\left[\begin{array}{c}
\left(1+\lambda_{i}\right) \cos \phi_{i}+\left(1+\lambda_{i+1}\right) \cos \phi_{i+1} \\
\left(1+\lambda_{i}\right) \sin \phi_{i}+\left(1+\lambda_{i+1}\right) \sin \phi_{i+1}
\end{array}\right],
$$

respectively. Here $\lambda$ is the radial and $\phi$ is the angular deviation from the relative positions in the ground state.

Substituting Eqs. (33) and (34) into Eq. (32), for small fluctuations, the electrostatic energy becomes 


$$
\begin{aligned}
\frac{U_{n}}{k_{B} T}= & \frac{1}{T^{*}}\left\{S(n)+\sum_{k=1}^{n-1} \lambda_{k}-\frac{1}{4} \sum_{k=1}^{n-2}\left[\lambda_{k}+\lambda_{k+1}\right.\right. \\
& \left.\left.-\frac{1}{4}\left(\phi_{k}-\phi_{k+1}\right)^{2}\right]\right\} .
\end{aligned}
$$

In the low-temperature limit, configurational integral, Eq. (28) can be performed explicitly yielding,

$$
\xi_{n}\left(T^{*}\right)=\left(\frac{a^{2 n-2}}{9}\right) 2^{2 n} \pi^{n / 2} T^{* 3 n / 2-2} e^{-S(n) / T^{*}} \text { for } n \geqslant 3 \text {. }
$$

The prefactor is the result of thermal fluctuations while the exponential is due to the ground-state energy.

The condition of chemical equilibrium between the monopoles and the $n$ chains is expressed through the law of mass action $\mu_{n}=t \mu_{+}+s \mu_{-}$. The chemical potential for monopoles is given by Eq. (19) with $\rho \rightarrow \rho_{1}$, while for $n$ chains the chemical potential is

$$
\mu_{n}=k_{B} T \ln \left[\frac{\rho_{n} \Lambda^{2 n}}{\xi_{n}(T)}\right]
$$

The law of mass action reduces to

$$
\rho_{n}=\left(\frac{\rho_{1}}{2}\right)^{n} \xi_{n}\left(T^{*}\right) e^{n \beta \mu_{ \pm}^{e x}}
$$

At the level of approximation that we have adopted, the chains are treated as noninteracting ideal species. This means that just like in the case of dipoles in Sec. III, they cannot affect location of the critical point. Therefore $T_{c}^{*}$ $=0.0517386$ and $\rho_{1 c}^{*}=0.00243975$. Substituting these values into Eq. (38) we find that the sum in Eq. (27) diverges. In fact according to the Cauchy criterion, the sum in Eq. (27) will converge absolutely, if and only if,

$$
\Delta \equiv \lim _{n \rightarrow \infty} \rho_{n}^{* 1 / n}<1
$$

Using Eq. (38),

$$
\Delta=2 \rho_{1}^{*} \sqrt{\pi T^{* 3}} \exp \left[\frac{\alpha+I(x) / \tau_{1}(x)}{2 T^{*}}\right],
$$

where $\alpha=2 \ln 2$ and $x=\kappa_{s}\left(\rho_{1}\right) a$. Inserting the critical parameters into the expression above, we find that at criticality $\Delta_{c}=5.82706$, and the Cauchy criterion is strongly violated. Therefore, the critical density of monopoles lies outside the radius of convergence of Eq. (27). This means that for any finite density $\rho$, the density of monopoles never reaches the threshold necessary for the phase separation to occur, $\rho_{1}$ $<\rho_{1 c}$.

\section{RESULTS AND DISCUSSION}

We have presented an argument that suggests that a confined $2 d$ electrolyte should not phase separate. Instead, just as for the case of dipolar hard spheres, as the temperature is lowered, the ions will associate forming polymerlike chains of alternating positive and negative monomers. Clearly our argument is based on a number of assumptions. First, we have supposed that in the critical region the linear chains predominate over the compact clusters. This is far from obvious. If the compact clusters have lower free energy than the chains, they can provide nuclei for the condensation and the gas-liquid phase separation. A second assumption implicit in our calculations is that the chains interact only weakly. This is somewhat easier to justify. Consider an infinitely long rigid line of alternating charges $(\cdots+-+-+$ $-\cdots)$. Suppose that the center to center distance between the nearest neighbors is $a$. It is then possible to show that the potential produced by such a line of charge decays exponentially, $\psi(r) \sim \exp (-\pi r / a)$, where $r$ is the distance perpendicular to the chain. Thus, the interactions between long polymerlike clusters should, indeed, be quite weak. However, the shorter chains can still interact sufficiently strongly to drive phase transition [20]. Finally, even if the formation of chains prevents the liquid-gas phase separation, it does not forestall other kinds of phase transitions from taking place [21]. At sufficiently high densities, the two component plasma will crystallize exhibiting a pseudolong-range order. At low temperatures and densities, where the polymerlike chains predominate, the $Y$-like defect formation [22] can lead to a coexistence between two phases, one with high and the other with low concentration of defects [23].

After this paper was completed and submitted for publication, the referee drew our attention to a recently published simulation by Weis, Levesque, and Caillol (WLC) [24] of a $2 d$ ionic fluid. Indeed, these authors found coexistence between high- and low-density phases. WLC estimated the critical temperature to be $T_{c} \approx 0.04$, which should be compared with our Debye-Hückel prediction of $T_{c}=0.052$. Furthermore, in the low-density phase, WLC found predominance of chain and ringlike clusters. The high-density phase resembles a percolating gellike cluster [24]. Although there is a phase coexistence, it is difficult to associate it with a traditional liquid-vapor transition. Instead the coexistence resembles more a sol-gel transition in polymer systems. The task for theorists must now be to quantitatively understand the phase transition found by WLC. We hope that the current paper will provide a first step in this direction.

\section{ACKNOWLEDGMENTS}

This work was supported by the Brazilian science agencies CNPq, FINEP, and Fapergs. J.E.F.M. acknowledges the financial support from the CNPq and CLAF during the development of this work, and partial financial support from CONACyT. M.C.B. is grateful for the hospitality at the Center of Polymer Studies at Boston University, where part of this work was done. 
[1] R.R. Singh and K.S. Pitzer, J. Chem. Phys. 92, 6775 (1990).

[2] H. Weingärtner et al., J. Chem. Phys. 96, 848 (1992); T. Narayanan and K.S. Pitzer, Phys. Rev. Lett. 73, 3002 (1994); W. Schröer et al., J. Phys.: Condens. Matter 8, 9329 (1996).

[3] M.E. Fisher and Y. Levin, Phys. Rev. Lett. 71, 3826 (1996).

[4] Y. Levin and M.E. Fisher, Physica A 225, 164 (1996).

[5] M.E. Fisher and B.P. Lee, Phys. Rev. Lett. 77, 3561 (1996).

[6] J. Valleau and G. Torrie, J. Chem. Phys. 108, 5169 (1998).

[7] J.J. Weis and D. Levesque, Phys. Rev. Lett. 71, 2729 (1993); J.-M. Caillol, J. Chem. Phys. 98, 9835 (1993); M.E. van Leeuwen and B. Smit, Phys. Rev. Lett. 71, 3991 (1993); M.J. Stevens and G.S. Grest, ibid. 72, 3686 (1994).

[8] R.P. Sear, Phys. Rev. Lett. 76, 2310 (1996).

[9] Y. Levin, Phys. Rev. Lett. 83, 1159 (1999).

[10] E.S. Velazquez and L. Blum, Physica A 244, 453 (1997).

[11] R.A. Salkar et al., J. Chem. Soc. Chem. Commun. 10, 1223 (1996).

[12] J.P. Valleau, J. Chem. Phys. 95, 584 (1991); A.Z. Panagiotopoulos, Fluid Phase Equilibria 76, 92 (1993); J.M. Cailol, J. Chem. Phys. 100, 2161 (1994); J.M. Cailol et al., ibid. 107,
1565 (1997); G. Orkoulas and A.Z. Panagiotopoulos, ibid. 110, 1581 (1999); Q. Yan and J.J. de Pablo, ibid. 111, 9509 (1999).

[13] Y. Levin, Physica A 265, 432 (1999).

[14] P.W. Debye and E. Hückel, Phys. Z. 24, 185 (1923); D.A. McQuarrie, Statistical Mechanics (Harper \& Row, New York, 1971), Chap. 15.

[15] Handbook of Mathematical Functions, edited by M. Abramowitz and I.A. Stegun (Dover, New York, 1968).

[16] E.Z. Güntelberg, Z. Phys. Chem. 123, 199 (1926).

[17] N. Bjerrum, Kgl. Dan. Vidensk. Selsk. Mat.-fys. Medd. 7, 1 (1926).

[18] W. Ebeling, Z. Phys. Chem. (Leipzig) 238, 400 (1968).

[19] I.S. Jacobs and C.P. Bean, Phys. Rev. 100, 1060 (1955).

[20] J.C. Shelley and G.N. Patey, J. Chem. Phys. 103, 8299 (1995).

[21] P.J. Camp, J.C. Shelley, and G.N. Patey, Phys. Rev. Lett. 84, 115 (2000).

[22] T. Tlusty and S.A. Safran, Science 290, 1328 (2000).

[23] P. Pincus, Science 290, 1307 (2000).

[24] J.J. Weis, D. Levesque, and J.M. Caillol, J. Chem. Phys. 109, 7486 (1998). 